\title{
The Unexpected Spectrum of the Innermost Ejecta of the Red Hypergiant VY CMa*
}

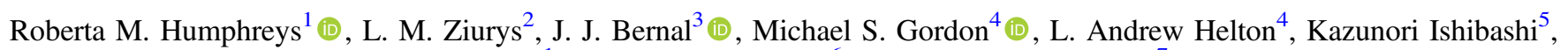 \\ Terry J. Jones $^{1}$ (D), A. M. S. Richards ${ }^{6}$, and Wouter Vlemmings ${ }^{7}$ (D) \\ ${ }^{1}$ Minnesota Institute for Astrophysics, University of Minnesota, Minneapolis, MN 55455, USA; roberta@umn.edu \\ ${ }^{2}$ Departments of Astronomy and Chemistry, University of Arizona, Tucson, AZ, USA; 1ziurys@email.arizona.edu \\ ${ }^{3}$ Department of Chemistry, University of Arizona, Tucson, AZ, USA \\ ${ }^{4}$ USRA-SOFIA Science Center, NASA Ames Research Center, Moffett Field, CA 94035, USA \\ ${ }^{5}$ Graduate School of Science, Nagoya University, Nagoya, 464-8602, Japan \\ ${ }^{6}$ Jodrell Bank, Department of Physics and Astronomy, University of Manchester, Manchester, UK \\ ${ }^{7}$ Department of Space, Earth and Environment, Chalmers University of Technology, Onsala Space Observatory, SE-439 92 Onsala, Sweden \\ Received 2019 January 29; revised 2019 March 19; accepted 2019 March 20; published 2019 April 3
}

\begin{abstract}
Hubble Space Telescope/STIS spectra of the small clumps and filaments closest to the central star in VY CMa reveal that the very strong $\mathrm{KI}$ emission and $\mathrm{TiO}$ and $\mathrm{VO}$ molecular emission, long thought to form in a dusty circumstellar shell, actually originate in a few small clumps hundreds of au from the star. The K I lines are 10-20 times stronger in these nearest ejecta than on the star. The observations also confirm VO as a circumstellar molecule. In this Letter we discuss the spectra of the features, their motions and ages, and the identification of the molecular emission. The strength of the atomic and molecular features in the small clumps present an astrophysical problem for the excitation process. We show that the clumps must have a nearly clear line of sight to the star's radiation.
\end{abstract}

Key words: circumstellar matter - ISM: molecules - line: identification - stars: individual (VY Canis Majoris) stars: massive - stars: mass-loss - stars: winds, outflows

\section{Introduction}

The red hypergiant VY CMa is the most extreme known case of stellar surface activity in terms of energy, mass outflow, and size scale. It is one of the strongest $10-20 \mu \mathrm{m}$ sources in the sky, with powerful maser activity, an unusual set of molecular features, the strongest known KI emission, and other rare characteristics. This unique combination of traits makes it one of the most important objects for understanding episodic mass loss and stellar activity. Unfortunately, ground-based spectra do not resolve the dust formation zone and the geometry of the inner ejecta. In this Letter we report Hubble Space Telescope (HST)/STIS spectroscopy with spatial resolution 0 ". 1 . Unexpectedly, these data reveal that molecular and $\mathrm{K}$ I emission are concentrated in a few bright knots, $\approx 0$ ". 3 from the star, rather than being distributed in a dusty circumstellar envelope.

Earlier multiwavelength HST/WFPC2 images revealed a circumstellar environment with numerous knots, arcs, and prominence-like loops visible by scattered light (Smith et al. 2001). Using transverse motions measured from second-epoch images plus ground-based Doppler velocities (Humphreys et al. 2005, hereafter Paper I), we mapped the 3D morphology of the ejecta and showed that these features were both spatially and kinematically distinct from the surrounding nebulosity (Humphreys et al. 2007, hereafter Paper II; Jones et al. 2007). They were ejected at different times over several hundred years in different directions, apparently by localized processes from different regions on the star.

Mass estimates from surface photometry in the HST images and from near- and mid-IR imaging and polarimetry of the SW Clump, yield minimum masses (dust + gas) of $>5 \times 10^{-3} M_{\odot}$ (Shenoy et al. 2013; Gordon et al. 2019). Atacama Large

\footnotetext{
* Based on observations made with the NASA/ESA Hubble Space Telescope which is operated by the Association of Universities for Research in Astronomy, Inc., under NASA contract NAS 5-26555.
}

Millimeter Array (ALMA) submillimeter observations reveal prominent dust components $\approx 0 . " 5$ from the star with the brightest (Clump C) having a total mass of at least $2.5 \times 10^{-3} M_{\odot}\left(\mathrm{O}^{\prime}\right.$ Gorman et al. 2015). More recent ALMA measurements of Clump $\mathrm{C}$ yield a dust mass alone of $>1.2 \times 10^{-3} M_{\odot}$, implying a total mass of $\sim 0.1 M_{\odot}$ (Vlemmings et al. 2017). These measurements imply short-term, high mass loss in separate events over several hundred years.

The mass-loss mechanism for red supergiants is not understood. Leading processes include radiation pressure on grains, pulsation, and convection, with convection being the most promising. The prominent arcs and dusty clumps in VY CMa provide clues to an additional mechanism, surface activity, and magnetic fields. The presence of magnetic fields is supported by the Zeeman splitting and polarization of the masers in its circumstellar ejecta (Vlemmings et al. 2002, 2003, 2005; Shinnaga et al. 2017). Recent ALMA continuum observations (Vlemmings et al. 2017) reveal polarized dust emission from magnetically aligned gains within 0.15 of the star.

In addition to the large-scale features clearly visible in the HST images, the ejecta are filled with numerous small condensations and filaments, suggesting that lesser events have occurred. Ground-based spectroscopy has been limited to 0 ". 8 spatial resolution (Paper I). Several small dusty knots and filaments $\leqslant 0$ ". 5 from the star very likely represent the most recent mass-loss events. We therefore obtained HST/STIS observations with a spatial resolution of 0 !" 1 to probe VY CMa's innermost ejecta and the frequency of recent events.

Our spectra of the star and the structures closest to the star yielded a surprising result. The very strong K I emission lines generated by resonant scattering and the molecular electronic spectra, assumed to form in the dusty envelope around the star, actually arise in the knots, not the immediate environment of the star. These observations also indicate that VO is actually a 


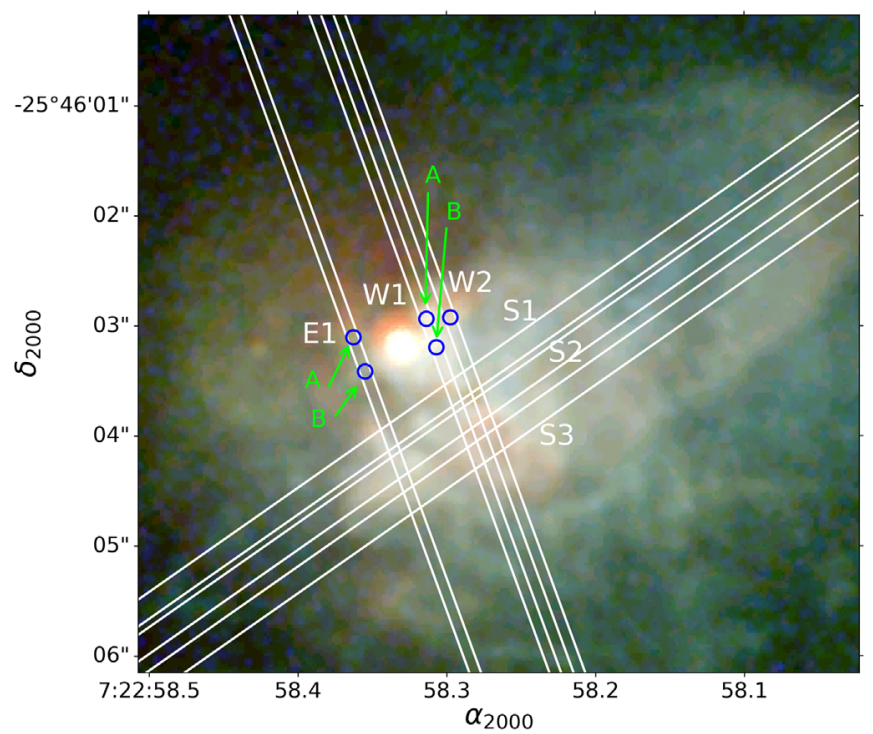

Figure 1. Slit positions for the HST/STIS spectra superposed on an HST image of VY CMa. The positions for the extractions discussed in the Letter are marked by circles.

circumstellar molecule. In this Letter we focus on the spectra of these knots and filaments nearest the star and the identification of the circumstellar molecular transitions. We also discuss the motions of the knots, their spatial orientation, and time since ejection, as well as the implications for the excitation of the atomic and molecular lines.

\section{HST/STIS Observations}

The HST/STIS observations were planned in two visits to allow for two different slit orientations to cover the small knots and filaments immediately to the west and east of the star with three slit positions and to separate the individual knots to the south and southwest of the star with three slit positions as shown in Figure 1. The central $\operatorname{star}^{8}$ was observed at each visit. Slits W1 and W2 were placed over the nebulous condensations visible in Figure 1. Although there are no obviously associated optical features with ALMA Clump C, slit E1 passed over the Clump $\mathrm{C}$ region and faint filaments just to the east of the star.

We used the STIS/CCD with the G750M grating at tilts 7795 and $8561 \AA$ to measure the K I emission lines at 7665 and $7699 \AA$ and the Ca II absorption triplet (8498, 8542, $8662 \AA$ ). The K I lines, formed by resonant scattering, are the strongest emission features in VY CMa and are the best tracers of the gas. The strong Ca II lines are reflected by dust. The $52 \times 0.1$ slit was used for the west/east knots and filaments and the 0 !" 2 slit was chosen for the knots south of the star to cover the somewhat more extended structures. At the $1.2 \mathrm{kpc}$ distance of VY CMa (Zhang et al. 2012), 0!"1 is $\approx 120$ au. After acquisition, the star was centered on the slit using a peakup in the red, and the telescope was then offset to the positions shown in Figure 1. The observations were completed on 2018 January 5 and February 11.

All of the spectra were processed using the subpixel modeling technique developed for the eta Car Treasury programs (Davidson 2006) to mitigate degradation of the $H S T$ 's spatial resolution along the slit. Consequently, the scale

\footnotetext{
The star is obscured by circumstellar dust, thus its spectrum is observed by scattered light. By "central star" we mean the bright object $r \lesssim 0.07$ arcsec.
}

in the processed spectra is 0 ". 0253 per pixel. Contemporary flat-field images were also obtained to correct for fringing and were processed in the same way as the science images and were normalized to be used as contemporary fringe flat-field images.

\section{Spectra and Motions of the Inner Knots}

We extracted one-dimensional spectra of the visible knots in slits W1 and W2 and at two positions in slit E1 as well as the star with a spatial width of 0 !. 1 along the slit. Sample extracted spectra are shown in Figure 2. The differences between the star and W1 knot A are immediately apparent. The molecular emission bands in the $7795 \AA$ spectrum and the broad molecular emission band and atomic emission lines in the $8561 \AA$ spectrum are unique to the three extractions west of the star. The spectra of knot B in W1 and the knot in W2 are very similar to knot A with small differences in the flux level of the continuum due to different distances from the star. The emission bands are not present in the two extractions in E1, nor are they present in any spectra extracted in slits to the south of the star.

The $\mathrm{KI}$ emission lines are much stronger relative to the continuum level in W1 knots A and B and the W2 knot than on the star. The total flux in the K I emission profiles is 10-20 times greater than on the star. All three knots show absorption components in the $\mathrm{K}$ I lines as does the star. The Rb I emission lines at 7800 and $7947 \AA$ (Wallerstein 1971) are not detectable in the spectrum of the star. The $8561 \AA$ spectra are dominated by the broad molecular feature and the strong Ca II triplet in absorption. Numerous weaker absorption lines are present due to Fe I and Ti I. The measured parameters of the K I emission lines, their Heliocentric velocities, and the velocities of the absorption lines are summarized in Table 1 for the star and at these five positions.

We measured the transverse motions of the these knots and filaments following the procedure described in Paper II using our two epochs of HST/WFPC2 images from 1999 and 2005. VY CMa was imaged in four filters, F410M, F547M, F656N, and F1042M, with a range of exposure times. The images are tightly aligned in pixel space and blinked to identify any offset. We measure the $x$ and $y$ positions of the blinked images three times in each filter combination in which the knot or filament is identified. The measurements are then combined for a weighted mean. In combination with the Doppler velocity measured from the K I lines relative to the star, we determine each clump's orientation, direction of motion, and total space velocity and then estimate its age or time since ejection included in Table 1.

The three small condensations to the west of the star are well defined, but the more filamentary features in slit E1 are more irregular and difficult to measure. We were not able to measure any reproducible positions for $\mathrm{B}$ in $\mathrm{E} 1$. The results for $\mathrm{E} 1$ position A are based on just the highest quality measurements in the F656 filter; see Paper II. It is not surprising that these knots and filaments closest to the star are the most recent ejections. They appear to fall into two time epochs of about 1920-1930 and 1900, which likely represent VY CMa's most recent active periods.

\section{The Molecular and Atomic Emission Features}

Numerous atomic emission lines and molecular bandheads are present in the spectrum of VY CMa. Many of the TiO and VO transitions were observed by Wallerstein $(1971,1986)$ and 

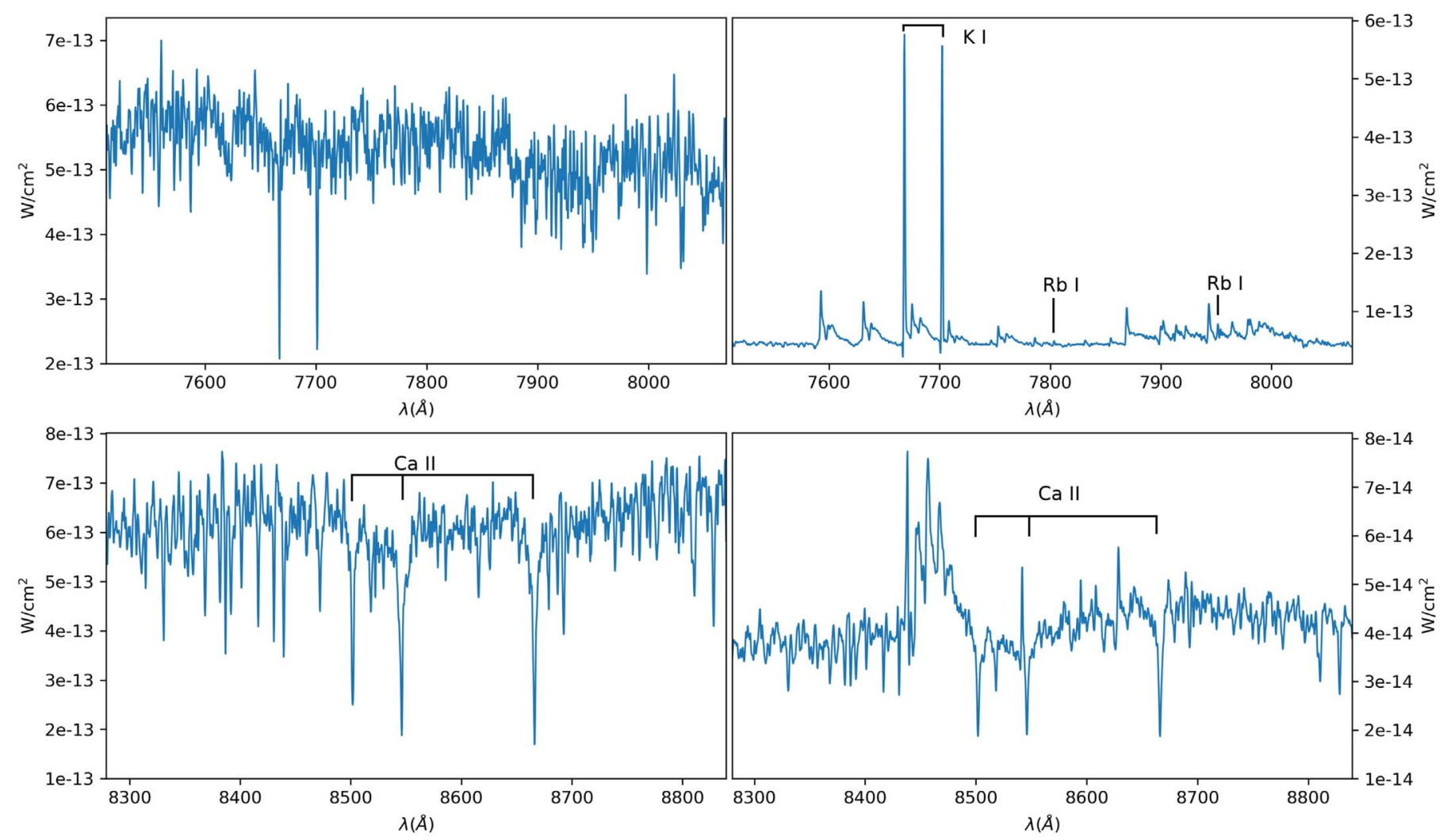

Figure 2. The two upper spectra are of the star on the left and knot A in the W1 slit on the right showing the strong K I emission lines and molecular bands. The bottom spectra are the star on the left and knot A in the W1 slit showing the Ca II triplet in each and the previously unrecognized strong molecular emission band and unidentified features in the knot.

Table 1

Measured Fluxes, Heliocentric Velocities, and Motions

\begin{tabular}{|c|c|c|c|c|c|c|}
\hline $\begin{array}{l}\text { Object } \\
\text { Name }\end{array}$ & & & $\begin{array}{c}\begin{array}{c}\text { K I em Vel } \\
\left(\mathrm{km} \mathrm{s}^{-1}\right)\end{array} \\
\end{array}$ & \multicolumn{2}{|c|}{$\begin{array}{c}\begin{array}{c}\text { K I abs Vel } \\
\left(\mathrm{km} \mathrm{s}^{-1}\right)\end{array}\end{array}$} & $\begin{array}{c}\text { Abs. Lines Vel. } \\
\left(\mathrm{km} \mathrm{s}^{-1}\right)\end{array}$ \\
\hline & \multicolumn{2}{|c|}{$6.7 \times 10^{-14}, 3.0 \times 10^{-14}$} & $50.8,59.2$ & \multicolumn{2}{|c|}{$9.0,4.7$} & $67.1(21)$ \\
\hline W1 knot A & \multicolumn{2}{|c|}{$7.2 \times 10^{-13}, 6.5 \times 10^{-13}$} & $42.6,44.0$ & \multicolumn{2}{|c|}{$-12.5,-10.9$} & $57.7(17)$ \\
\hline W1 knot B & \multicolumn{2}{|c|}{$8.1 \times 10^{-13}, 8.4 \times 10^{-13}$} & $45.8,46.0$ & \multicolumn{2}{|c|}{$-16.4, \cdots$} & $67.7(15)$ \\
\hline W2 knot & \multicolumn{2}{|c|}{$5.0 \times 10^{-13}, 5.2 \times 10^{-13}$} & $47.3,46.0$ & \multicolumn{2}{|c|}{$-22,-24.5$} & $76.0(13)$ \\
\hline E1 A & \multicolumn{2}{|c|}{$6.1 \times 10^{-15}, 1.1 \times 10^{-14}$} & $45.4,45.2$ & \multicolumn{2}{|c|}{$0.4,-1.5$} & $81.5(15)$ \\
\hline E1 B & \multicolumn{2}{|c|}{$5.3 \times 10^{-15}, 6.5 \times 10^{-15}$} & $47.3,44.8$ & \multicolumn{2}{|c|}{$-5,-3.9$} & $\cdots$ \\
\hline $\begin{array}{l}\text { Object } \\
\text { Name }\end{array}$ & $\begin{array}{c}\text { Trans Vel. } \\
\left(\mathrm{km} \mathrm{s}^{-1}\right)\end{array}$ & $\begin{array}{l}\text { Direct. } \phi \\
\quad(\operatorname{deg})\end{array}$ & $\begin{array}{l}\text { Orient. } \theta \\
\text { (deg) }\end{array}$ & $\begin{array}{l}\text { Total Vel. } \\
\left(\mathrm{km} \mathrm{s}^{-1}\right)\end{array}$ & $\begin{array}{l}\text { Dist. } \\
\text { (au) }\end{array}$ & $\begin{array}{l}\text { Age } \\
(\mathrm{yr})\end{array}$ \\
\hline Star & $\ldots$ & $\ldots$ & $\ldots$ & $\ldots$ & $\ldots$ & $\cdots$ \\
\hline W1 knot A & $21.6 \pm 4$ & $-45.6 \pm 6$ & $-28 \pm 5$ & $24.6 \pm 4.5$ & 390 & $77 \pm 14(1922)$ \\
\hline W1 knot B & $18.9 \pm 6$ & $-14 \pm 12$ & $-25 \pm 6$ & $21.5 \pm 6$ & 300 & $67 \pm 20(1932)$ \\
\hline W2 knot & $32.6 \pm 10$ & $-43 \pm 10$ & $-15 \pm 5$ & $32.6 \pm 10$ & 640 & $94 \pm 28(1905)$ \\
\hline E1 A & $27.4 \pm 5$ & $50 \pm 8$ & $-19 \pm 4$ & $29.0 \pm 6$ & 590 & $98 \pm 20(1901)$ \\
\hline E1 B & $\ldots$ & $\ldots$ & $\ldots$ & $\ldots$ & $\ldots$ & $\ldots$ \\
\hline
\end{tabular}

Note.

a The fluxes here are measured in a 0 ! $1 \times 0$ ! 1 aperture. The clumps are somewhat larger.

Wallerstein \& Gonzalez (2001). A bandhead of $\mathrm{ScO}$ was also identified by Wallerstein (cited in Hyland et al. 1969) and by Herbig (1974). We confirm many of these past identifications, as well as new molecular bands, including the broad feature in the far-red spectrum. We also demonstrate that these molecules are clearly circumstellar, as opposed to being formed closer to the star in its wind. We thus present the first conclusive evidence for the identification of $\mathrm{VO}$ as circumstellar. Our identifications are summarized in Table 2 and shown in Figure 3.

We identify VO bandheads of the $B^{4} \Pi-X^{4} \Sigma$ electronic transition. Figure 3 shows spectra of the $(0,0)$ and $(0,1)$ vibrational transitions. A simulation of the $(0,0)$ spectra is also shown above the astronomical data, using pGopher (Western 2014). The simulation reproduces the observed spectrum quite well, assuming a temperature of $\sim 450 \mathrm{~K}$. The complexity of the spectrum arises from the presence of fine structure and $P$ - 
Table 2

Molecular Emission and Atomic Emission Line Identifications

\begin{tabular}{|c|c|c|c|}
\hline $\begin{array}{l}\text { Vacuum Wave- } \\
\text { length } \AA\end{array}$ & $\begin{array}{l}\text { Lab Vacuum } \\
\text { Wavelength } \AA\end{array}$ & Identification & References \\
\hline 7592.8 & $7591.5^{\mathrm{a}}$ & $\begin{array}{c}\text { TiO A-X } R_{3} \\
(0,1)\end{array}$ & $(1),(2)$ \\
\hline 7631.0 & $7629.9^{\mathrm{a}}$ & $\begin{array}{l}\mathrm{TiO} \mathrm{A}-\mathrm{X} R_{2} \\
\quad(0,1)\end{array}$ & This work, (2) \\
\hline 7668.1 & 7667.0 & K I & (1) \\
\hline 7675.1 & $7673.7^{\mathrm{a}}$ & $\begin{array}{l}\mathrm{TiO} \mathrm{A}-\mathrm{X} R_{1} \\
\quad(0,1)\end{array}$ & (1), (2) \\
\hline 7702.23 & 7701.10 & K I & (1) \\
\hline 7708.4 & $7707.1^{\mathrm{a}}$ & $\mathrm{TiO} \mathrm{A}-\mathrm{X}_{2}$ & $(1),(2)$ \\
\hline 7753.0 & $7751.6^{\mathrm{a}}$ & $\begin{array}{c}\mathrm{TiO} \mathrm{A}-\mathrm{X} R_{1} \\
\quad(1,2)\end{array}$ & This work, (2) \\
\hline 7786.0 & $7785.0^{\mathrm{a}}$ & $\begin{array}{l}\mathrm{TiO} \mathrm{A}-\mathrm{X} R_{2} \\
\quad(2,3)\end{array}$ & (2), (3) \\
\hline 7803.4 & 7802.38 & $\mathrm{Rb} \mathrm{I}$ & (1) \\
\hline 7831.7 & $7830.3^{\mathrm{a}}$ & $\begin{array}{l}\text { TiO A-X } R_{1} \\
\quad(2,3)\end{array}$ & (2), (3) \\
\hline 7854.7 & $7853.2^{\mathrm{a}}$ & VO B-X $(0,0)$ & $\begin{array}{l}\text { This work, } \\
\text { (4), (5) }\end{array}$ \\
\hline 7869.2 & $\sim 7867^{\mathrm{b}}$ & VO B-X $(0,0)$ & $(1),(4),(5)$ \\
\hline $7901.3^{\mathrm{c}}$ & $\sim 7899^{\mathrm{b}}$ & VO B-X $(0,0)$ & $\begin{array}{l}\text { This work, } \\
\text { (4), (5) }\end{array}$ \\
\hline 7913.8 & $7912.3^{\mathrm{d}}$ & VO B-X $(0,0)$ & $(1),(4),(5)$ \\
\hline 7922.7 & $\sim 7920^{\mathrm{b}}$ & VO B-X $(0,0)$ & $\begin{array}{l}\text { This work, } \\
\text { (4), (5) }\end{array}$ \\
\hline 7943.4 & $\sim 7940^{\mathrm{b}}$ & VO B-X $(0,0)$ & $(1),(4),(5)$ \\
\hline $7951.5^{\mathrm{c}}$ & 7949.8 & $\mathrm{RbI}+\mathrm{VO}^{\mathrm{c}}$ & (1) \\
\hline 7964.5 & $\sim 7963^{\mathrm{b}}$ & VO B-X $(1,1)$ & $\begin{array}{l}\text { This work, } \\
\text { (4), (5) }\end{array}$ \\
\hline $7979.8^{\mathrm{c}}$ & $\sim 7978^{\mathrm{b}}$ & VO B-X $(1,1)$ & $\begin{array}{l}\text { This work, } \\
\text { (4), (5) }\end{array}$ \\
\hline 7988.9 & $\sim 7987^{\mathrm{b}}$ & VO B-X $(1,1)$ & $\begin{array}{l}\text { This work, } \\
(4),(5)\end{array}$ \\
\hline 8438.15 & $\ldots$ & unknown & $\ldots$ \\
\hline 8448.2 & $8446.4^{\mathrm{e}}$ & TiO E-X $(0,0)$ & This work, (6) \\
\hline 8456.8 & $8455.6^{\mathrm{e}}$ & TiO E-X $(0,0)$ & This work, (6) \\
\hline 8467.2 & $8466.1^{\mathrm{e}}$ & TiO E-X $(0,0)$ & This work, (6) \\
\hline 8476.7 & $\sim 8475^{\mathrm{b}}$ & TiO E-X $(0,0)$ & This work, (6) \\
\hline 8541.8 & $\sim 8540^{\mathrm{b}}$ & VO B-X $(0,1)$ & $\begin{array}{l}\text { This work, } \\
(4),(5)\end{array}$ \\
\hline 8594.6 & $\sim 8593^{\mathrm{b}}$ & VO B-X $(0,1)$ & $\begin{array}{l}\text { This work, } \\
\text { (4), (5) }\end{array}$ \\
\hline 8608.2 & $\sim 8607^{\mathrm{b}}$ & VO B-X $(0,1)$ & $\begin{array}{l}\text { This work, } \\
\text { (4), (5) }\end{array}$ \\
\hline 8628.7 & $\sim 8627^{\mathrm{b}}$ & VO B-X $(0,1)$ & $\begin{array}{l}\text { This work, } \\
(4),(5)\end{array}$ \\
\hline
\end{tabular}

Notes.

${ }^{\text {a }}$ Approximate $R$-branch bandhead.

b Blended branches.

${ }^{c}$ Blended feature.

${ }^{\mathrm{d}} Q$-branch bandhead.

${ }^{\mathrm{e}}$ Estimate of $Q$-branch maximum.

References. (1) Wallerstein (1971), (2) Ram et al. (1999), (3) Wallerstein \& Gonzalez (2001), (4) Keenan \& Schroeder (1952), (5) Adam et al. (1995), (6) Simard \& Hackett (1991).

, $Q$-, and $R$-branch line blending in the quartet-quartet electronic transition. Wallerstein (1971) identified some of these features (Table 3), but most are new assignments, based on the laboratory work of Keenan \& Schroeder (1952) and Adam et al. (1995). Cheung et al. (1994) and Adam et al. (1995) conducted the first accurate spectral analysis of VO, and made assignments of the fine-structure components unknown to Wallerstein. These spectra are definitive evidence for the presence of $\mathrm{VO}$ in circumstellar ejecta hundreds of au from the central star.

For $\mathrm{TiO}$, the $\mathrm{A}^{3} \Phi-\mathrm{X}^{3} \Delta$ electronic band is prominent with numerous sharp $R$-branch $(\Delta J=+1)$ bandheads with less intense $P$ and $Q$ features arising from the $(0,1),(1,2)$, and $(2$, 3 ) vibrational sequence. New identifications for $\mathrm{TiO}$ spectra are the $R_{2}(0,1)$ and $R_{1}(1,2)$ features, which are based on laboratory measurements of Ram et al. (1999). (The subscript on the $R$ refers to the spin-orbit component, as indicated by quantum number $\Omega$, of the lower level, where $\Omega=1,2$, and 3.) A simulation of the three fine-structure $(0,1)$ features is presented above the data, and reproduces the observed spectra well. The simulation assumes a temperature of $\sim 350 \mathrm{~K}$.

In addition to the $A-X$ electronic transition, the $E^{3} \Pi-X^{3} \Delta$ transition of the $(0,0)$ vibrational band of $\mathrm{TiO}$ is observed for the first time in VY CMa. As measured in the laboratory by Simard \& Hackett (1991), this band consists of four prominent peaks, arising from strong $Q$-branches blended with $P$ - and $R$ branch transitions, as shown by the simulation above the observed data. The E-X transition has been previously reported in the peculiar red nova merger remnants V4332 Sgr and V1309 Sco (Kaminski et al. 2015; Tylenda et al. 2015). The TiO E-X spectrum was best reproduced assuming $T \sim 250 \mathrm{~K}$. Note that the excited A and E states lie 13,000 and $11,800 \mathrm{~cm}^{-1}$ higher in energy above the ground states. Despite its designation, the $\mathrm{E}$ state actually lies lower in energy by about $1250 \mathrm{~cm}^{-1}$.

$\mathrm{TiO}_{2}$ emission has been mapped within $1^{\prime \prime}$ around the central star by de Beck et al. (2015) from observations with ALMA with $\sim 0$ !' 2 resolution. The morphology of the $\mathrm{TiO}_{2}$ emission with LSR velocities greater than $44 \mathrm{~km} \mathrm{~s}^{-1}$ overlaps the strong $\mathrm{TiO}$ (and K I) emission in the knots to the west of the star. The TiO A-X bandheads in W1 knot A have a mean Heliocentric velocity of $53.4 \pm 6.0,68 \mathrm{~km} \mathrm{~s}^{-1}$ LSR. This spatial and kinematic overlap suggests that the $\mathrm{TiO}_{2}$ emission may originate from the same dusty condensations. But a comparable correspondence does not apply to the region just east of the star. The velocities of the $\mathrm{TiO}_{2}$ emission there are blueshifted with an LSR velocity range of $28-43 \mathrm{~km} \mathrm{~s}^{-1}$ compared to the LSR velocity $\left(\sim 60 \mathrm{~km} \mathrm{~s}^{-1}\right)$ of the visible filaments to the east, but their spectra lack $\mathrm{TiO}$ emission.

One feature near $8438 \AA$ remains unidentified. The line profile is quite sharp, suggesting an atomic origin. However, Herbig (1974) explained how a complex structure in an electronic transition could be shrunk down to a single apparent line in VY CMa due to the crowding of the rotational structure near the bandheads. Possible wavelength agreements with Sc I and $\mathrm{Ca}$ I/Ca I] all involve unlikely high level transitions.

\section{A Basic Astrophysical Question-Excitation of the Strong K I and Molecular Emission in the Ejecta}

Evidently the extraordinary $\mathrm{KI}$ doublet and the strong molecular emission originate primarily in a few small diffuse clumps 0 ". 3 west of the central star, unresolved in groundbased spectra. Our results significantly alter the existing 

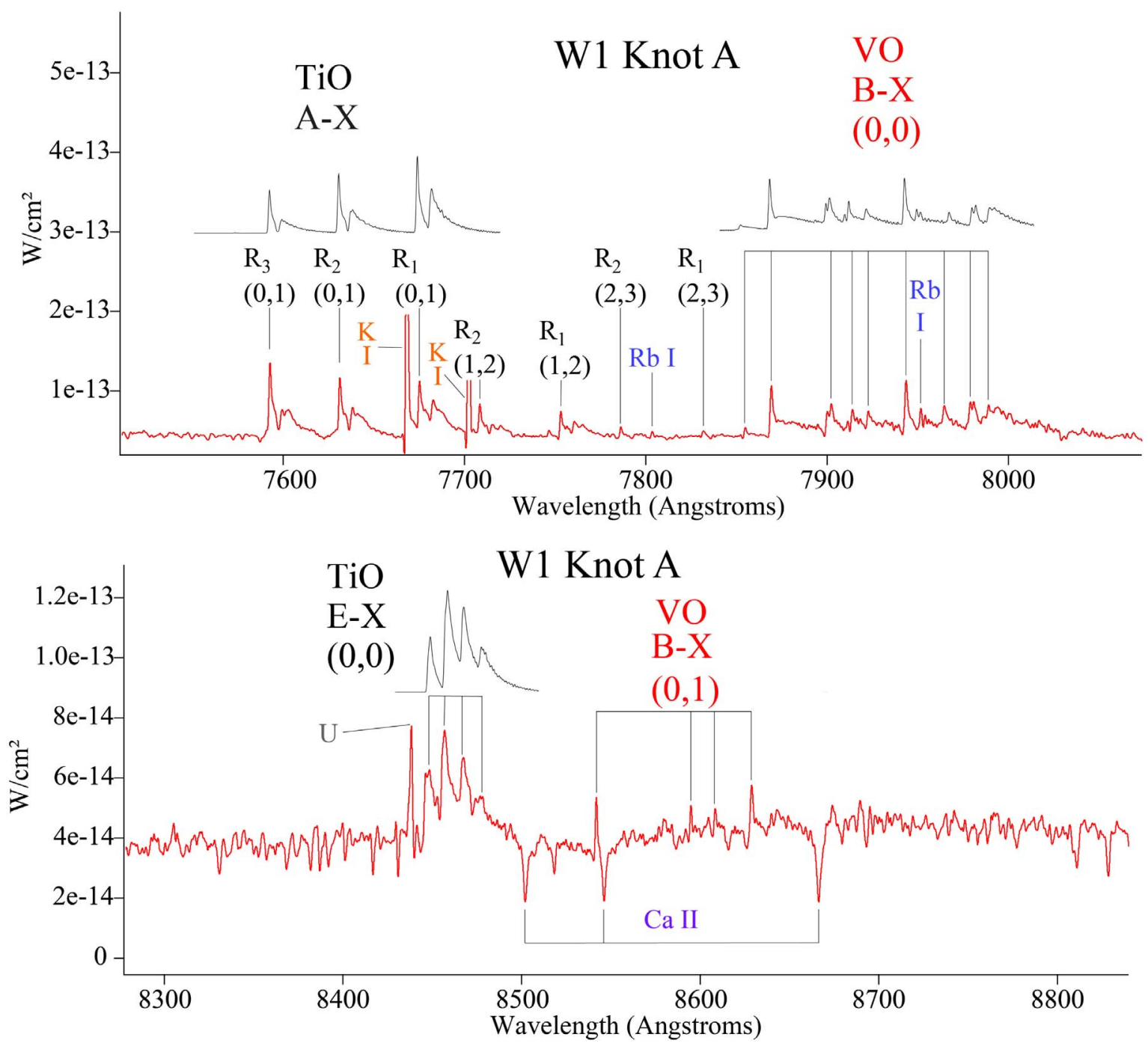

Figure 3. Molecular identifications from Table 2 shown on the red and far-red spectra. Simulations are shown above the stronger transitions. The K I lines are truncated.

models. In this Letter, we offer only a simplified outline of the problem. ${ }^{9}$

$\mathrm{KI}$ is observed throughout the the extended ejecta via resonant scattering (Paper I), but the strong molecular emission is observed only in the clumps to the west of the star. As our discussion below suggests, radiative excitation and resonant scattering are feasible for the strong K I doublet in the clumps. On the other hand, gas temperatures are probably important for the molecular emission. Our derived temperatures of 300-400 $\mathrm{K}$ are close to the expected dust-grain temperatures and radiation-density temperatures in the clumps. Excitation by shocks or MHD waves may be important for the molecules, but shocks have at least one disadvantage: the observed temperatures require low shock speeds of the order of $4 \mathrm{~km} \mathrm{~s}^{-1}$, inefficient for energy transport. Thus, our measurements support radiative excitation of the molecules that originate in the clumps, but collisional process may play a role.

The K I doublet is VY CMa's strongest emission feature, and it leads to a remarkable unpredicted result described below.

\footnotetext{
9 We do not discuss various semi-obvious caveats-e.g., the dependence on viewing direction, whether brightness might not imply a maximum in the gas density, etc.
}

With a total luminosity of the order of $4 L_{\odot}$ in just two narrow lines (Paper I), it greatly exceeds the KI features known in other evolved cool stars (Bernat \& Lambert 1976; Plez \& Lambert 1994; Guilain \& Mauron 1996). As explained in Paper I, resonance scattering of continuum photons is the only likely production mechanism. Paper I emphasized that this process is a zero-sum game; we must explain why the spectrum has no strong absorption feature comparable to the observed emission-i.e., like the absorption part of a pure-scattering $\mathrm{P}$ Cygni profile. In Appendix A2 of Paper I, we explained how the absorption can be hidden if the dust formation zone at $r \gtrsim 80$ au is very inhomogeneous. That scenario is too lengthy to repeat here, but a crucial point is that most escaping photons - roughly $2 \%$ of the star's output in the relevant wavelength range-follow multiply scattered paths between opaque condensations. In the simplest viable model, emergent KI would have a mottled appearance with a diameter of the order of 600 au ( $~ 500$ mas).

However, the HST/STIS data now show that most of the K I emission comes from a few condensations-for instance, Knot W1-A produces roughly $10 \%$ of the total K I flux associated with the "star image" in ground-based spectra (Paper I). Relatively little KI emission appears in the central object 
( $r \lesssim 80$ mas or $100 \mathrm{au}$ ). In the only model that appears realistic to us, the small clumps must "see" remarkably clear holes in the circumstellar dust shell. We expect to publish a detailed analysis later, and here we only sketch the reasoning for W1 knot A. (1) With a diameter of 200 au or less, located 400 au from the star, the knot intercepts $1 \%-2 \%$ of the star's light if there is no dust between them. (2) With $L \sim 3 \times 10^{5} L_{\odot}$ and $T \sim 3500 \mathrm{~K}$, the star radiates about $25 L \odot$ per $\AA$ around $7700 \AA$, so the knot intercepts $0.5 L_{\odot}$ per $\AA$ or less. (3) Each of the K I lines from knot W1-A has a luminosity of roughly $0.3 L_{\odot}$, corrected for interstellar extinction. (4) Therefore, if other conditions are favorable, then the knot's observed K I brightness can be attained if the incident continuum in wavelength interval $\Delta \lambda \gtrsim 0.6 \AA$ is converted via resonance scattering in the knot, required for the two K I lines. The corresponding velocity width is of the order of $30 \mathrm{~km} \mathrm{~s}^{-1}$, which appears possible with an allowable velocity dispersion in a knot and the K I oscillator strengths. The main result of this exercise is that the path from the star to each bright knot must be nearly free of dust; a 50\% extinction loss would increase the needed $\Delta \lambda$ to an implausible value.

This is a remarkable result, as dust is expected to have optical depths $\tau \sim 50$ along typical radial paths (Paper I). According to the above analysis, $\tau \lesssim 0.5$ in a roughly $30^{\circ}$-wide cone toward each bright knot. Thus, during roughly a century since the knots were ejected, the star ejected practically no material in those directions-even though it presumably rotates. Strong KI have been reported since 1958 (Wallerstein 1958). We suspect that this may be explained in terms of the statistics of size scales and timescales in the outflow structure, but it was not expected, and it gives an unpredicted constraint on the outflow physics.

As we emphasized earlier, the mass-loss mechanisms for RSGs are uncertain, and VY CMa is arguably the most significant example because it is extreme in several respects. Its localized sources of molecular emission and scattered atomic lines, reported above, were not predicted, and they imply major gaps or holes in the outflow structures formed by large-scale stellar activity. Related processes may occur in more normal RSGs. High-resolution imaging of $\alpha$ Ori has revealed small condensations and filaments within $1^{\prime \prime}$ (Kervella et al. 2011). Future theoretical models of active regions must account for these observed density contrasts and size scales.

R.H. thanks Kris Davidson and George Wallerstein for useful comments and discussion. This work was supported by NASA through grant GO-15076 (PI: R. Humphreys) from the Space Telescope Science Institute and National Science Foundation grant AST-1515568 (PI: L. Ziurys).

Facility: HST (STIS).

\section{ORCID iDs}

Roberta M. Humphreys (ib https://orcid.org/0000-00031720-9807

J. J. Bernal (iD https:// orcid.org/0000-0001-6176-0773

Michael S. Gordon (10 https://orcid.org/0000-0002-1913-2682

Terry J. Jones (iD https://orcid.org/0000-0002-8716-6980

Wouter Vlemmings (iD https://orcid.org/0000-0002-2700-9916

\section{References}

Adam, A. G., Barnes, M., Berno, B., et al. 1995, JMoSp, 170, 94

Allard, F, Hauschildt, P. H., \& Schwenke, D. 2000, ApJ, 540, 1005

Bernat, A. P., \& Lambert, D. L. 1976, ApJ, 210, 395

Cheung, A. S.-C., Hajigeorgious, P. G., Huanh, G., Huang, S.-Z., \& Merer, A. J. 1994, JMoSp, 163, 443

Davidson, K. 2006, in The 2005 HST Calibration Workshop: Hubble After the Transition to Two-gyro Mode, Proc. Workshop, ed. A. M. Koekemoer, P. Goudfrooij, \& L. L. Dressel (Baltimore, MD: STScI), 247

de Beck, E., Vlemmings, W., Muller, S., et al. 2015, A\&A, 580, A36

Dobrodey, N. V. 2001, A\&A, 365, 642

Gordon, M. S., Jones, T. J., Humphreys, R. M., et al. 2019, AJ, 157, 57

Guilain, Ch., \& Mauron, N. 1996, A\&A, 314, 585

Herbig, G. H. 1974, ApJ, 188, 533

Humphreys, R. M., Davidson, K., Ruch, G., \& Wallerstein, G. 2005, AJ, 129,492

Humphreys, R. M., Helton, L. A., \& Jones, T. J. 2007, AJ, 133, 2716

Hyland, E. E., Neugebauer, G., \& Wallerstain, G. 1969, ApJ, 158, 619

Jones, T. J., Humphreys, R. M., Helton, L. A., et al. 2007, AJ, 133, 2730

Kaminski, T., Tylenda, R., \& Schmidt, M. R. 2015, A\&A, 580, A34

Keenan, P. C., \& Schroeder, L. W. 1952, ApJ, 115, 82

Kervella, P., Perrin, G., Chiavassa, A., et al. 2011, A\&A, 531A, 117

Langhoff, S. R. 1997, ApJ, 481, 1007

O’Gorman, E., Vlemmings, W., Richards, A. M. S., et al. 2015, A\&A, 573, L1

Plez, B., \& Lambert, D. L. 1994, ApJL, 425, L101

Ram, R. S., Bernath, P. F., Dulick, M., \& Wallace, L. 1999, ApJS, 122, 331

Shenoy, D., Jones, T. J., Humphreys, R. M., et al. 2013, AJ, 146, 90

Shinnaga, H., Claussen, M. J., Yamamoto, S., \& Shimojo, M. 2017, PASJ, 69, L10

Simard, B., \& Hackett, P. A. 1991, JMoSp, 148, 128

Smith, N., Humphreys, R. M., Davidson, K., et al. 2001, AJ, 121, 1111

Tylenda, R., Górny, S. K., Kamiński, T., \& Schmidt, M. 2015, A\&A, 578A, 75

Vlemmings, W. H. T., Diamond, P. J., \& van Langevelde, H. J. 2003, ASSL, 283, 291

Vlemmings, W. H. T., Khouri, T., Marti-Vidal, I., et al. 2017, A\&A, 603, 92

Vlemmings, W. H. T., van Langevelde, H. J., \& Diamond, P. J. 2002, A\&A, 394, 589

Vlemmings, W. H. T., van Langevelde, H. J., \& Diamond, P. J. 2005, A\&A, 434, 1029

Wallerstein, G. 1958, PASP, 70, 479

Wallerstein, G. 1971, AJ, 169, 195

Wallerstein, G. 1986, A\&A, 164, 101

Wallerstein, G., \& Gonzalez, G. 2001, PASP, 113, 954

Western, C. M. 2014, PGOPHER Version 10.1, http://pgopher.chm.bris.ac. uk/Help/main.htm

Zhang, B., Reid, M. J., Menten, K. M., \& Zheng, X. W. 2012, ApJ, 744, 23 